\title{
Tensión Creativa aplicada al Análisis de Competencias a Alumnos de Ingeniería
}

\author{
Manuel Alcalà , Fernando Julián, Francisco J. Espinach, Narcís Verdaguer \\ Universitat de Girona, Escola Politecnica Superior, Dep. d'Organització, Gestió Empresarial i \\ Disseny de Producte, Campus Montilivi, 17071, Girona-España. (e-mail: manuel.alcala@udg.edu, \\ fernando.julian@udg.edu, francisco.espinach@udg.edu, narcis.verdaguer@udg.edu )
}

Recibido May. 14, 2010; Aceptado Jul. 01, 2010; Versión final recibida Jul. 14, 2010

\section{Resumen}

Este artículo refleja el análisis de competencias personales y sociales a partir del estudio de la tensión creativa en estudiantes de ingeniería, utilizando una aplicación informática denominada Cycloid. El objetivo se centra en comparar la tensión creativa de los estudiantes, actuando éstos como jefes de proyecto, siendo su carrera universitaria el proyecto a coordinar. El proceso consiste en evaluar, mediante encuestas, a un grupo de estudiantes para conocer la situación actual de las competencias utilizando lógica difusa. A partir del auto-conocimiento que brinda la herramienta, los alumnos pueden conocer sus puntos fuertes y débiles y gestionar mejor sus estudios. Los resultados obtenidos demuestran que la tolerancia al estrés y los idiomas son los puntos más débiles. Esta aplicación es útil en el diseño propio de su forma de trabajo que haga el estudiante y así desarrollar estrategias para afrontar con éxito sus estudios.

Palabras Clave: competencias, tensión creativa, ingeniería, gestión de proyectos, autoconocimiento

\section{Creative Tension Applied to the Analysis of Competences to Engineering Students.}

\begin{abstract}
This article reflects the analysis of personal and social competences through the study and analysis of creative tension in engineering students, using a computer application called Cycloid. The main objective was to compare the students' creative tension by asigning them the task of being the project leader of a given project: their own university major. The process consisted of evaluating, through special surveys, a group of students to know the current situation of competences, using fuzzy logic analysis. From this self-knowledge, provided by the survey, students can know their strong and weak characteristics regarding their study habits. Results showed that tolerance to stress and to language courses are the weaker points. This application is useful for the design of study strategies that students themselves can do to better face their courses.
\end{abstract}




\section{INTRODUCCIÓN}

La necesidad de comprobar cuáles son las competencias en las que nuestros alumnos destacan o en las que no, es un requisito fundamental por la importancia que tienen éstas en el ámbito del Espacio Europeo de Educación Superior (EEES). En nuestro caso hemos analizado las competencias transversales, utilizando una herramienta (TIC) Ilamada Cycloid, cuyo servidor se encuentra en Finlandia y que permite evaluar 30 competencias personales y sociales. Para ello, esta herramienta las estructura en seis grupos principales: conocimiento de uno mismo, autocontrol, capacidad cognitiva, auto motivación, empatía y habilidades sociales.

Nuestro grupo trabaja con esta herramienta desde el curso 2005-06. Este modelo identifica un trabajo genérico en gestión de proyectos y analiza la capacidad que tiene una persona para planificar, organizar, dirigir y evaluar trabajos complejos o proyectos que requieren una interacción sólida y frecuente con un equipo humano, con el fin de planificar todo el proyecto (Müller y Turner, 2010). En estas circunstancias, pueden evaluar sus estudios (su proyecto), llegando a autogestionarlos. Hemos realizado el estudio con alumnos de primer curso de Ingeniería de dos especialidades: los estudios de Ingeniería del diseño industrial y desarrollo de producto (DI) y los de Ingeniería en industrias agroalimentarias y explotaciones agropecuarias (EA), los cursos $2007 / 08$ y 2008/09. Nuestra opinión es que el modelo Cycloid mantiene un encaje importante con las competencias transversales de la Universidad de Girona (UdG).

EI EEES armoniza los sistemas nacionales de titulaciones, estableciendo un sistema europeo de créditos con la finalidad de comparar los estudios, favoreciendo la movilidad y creando un documento personalizado que incluye los estudios cursados, el contexto en que se han impartido y las competencias y capacidades profesionales que el alumno ha adquirido. Ante esta nueva situación, se ha evaluado en algunos informes el impacto de los cambios que se están produciendo. Uno de ellos es el elaborado por la Asociación de Universidades Europeas, el informe Trends IV (Reichert y Tauch, 2003), donde deja constancia de cómo el conocimiento de las competencias se considera una herramienta útil, en donde hemos pasado por tanto del aprendizaje de conocimientos al aprendizaje de éstas. Ahora, el estudiante debe acreditar el desarrollo de competencias que son potencialmente útiles en las actividades profesionales y en la sociedad en general (Coll y Zegwaard, 2006).

La UdG incluye cuatro bloques de competencias: las singulares, las transversales, las específicas y las específicas de cada asignatura. Dentro de las singulares, es decir, las que diferencian nuestra universidad, encontramos dos. La primera conecta la formación que proporciona la misma universidad con el entorno de la sociedad de Girona, recogiendo el compromiso de la misma con su entorno. La segunda establece que los estudiantes deben ser competentes para comparar e identificar las propias capacidades adquiridas durante los estudios, las necesidades del mercado profesional de Girona, así como la finalidad de reconocer posibles puestos de trabajo. Se pretende enlazar la formación con las necesidades del mercado laboral (IPM, 2006; Chang, 2009).

Nuestro estudio se centra en la autoevaluación de competencias y la comparación con el nivel que el estudiante considera debe tener. La aplicación trabaja con 30 competencias transversales que tienen una importante relación con las escogidas por nuestra universidad. Cycloid evalúa las competencias en gestión de proyectos, analizándolas por medio del proceso de autoevaluación. En nuestro caso, entendemos como proyecto la realización de los estudios universitarios de los alumnos. El sistema está basado en la arquitectura Evolute que se puede encontrar en Kantola et al., (2005) y Vanharanta (2005). En la figura 1 se describe el modelo en el que se muestra la teoría y las distintas aplicaciones desarrolladas. Las competencias se presentan categorizadas para evaluar características de las personas, en aspectos tales como: la selección de personal, la definición de programas de formación o de desarrollo, la planificación de carreras laborales, el análisis de la flexibilidad de las personas y otros El objetivo último es el diagnóstico y desarrollo de las personas para contribuir a la mejora de la competitividad de la organización (Mäkiniemi et al., 2004), o para llevar a cabo los objetivos formativos de una institución docente (Makatsoris, 2009). Un modelo de competencias debe recoger las necesarias para un puesto de trabajo concreto (Mäkiniemi et al., 2004; IPM, 2006) en nuestro caso el aprovechamiento positivo de los estudios del alumno. 


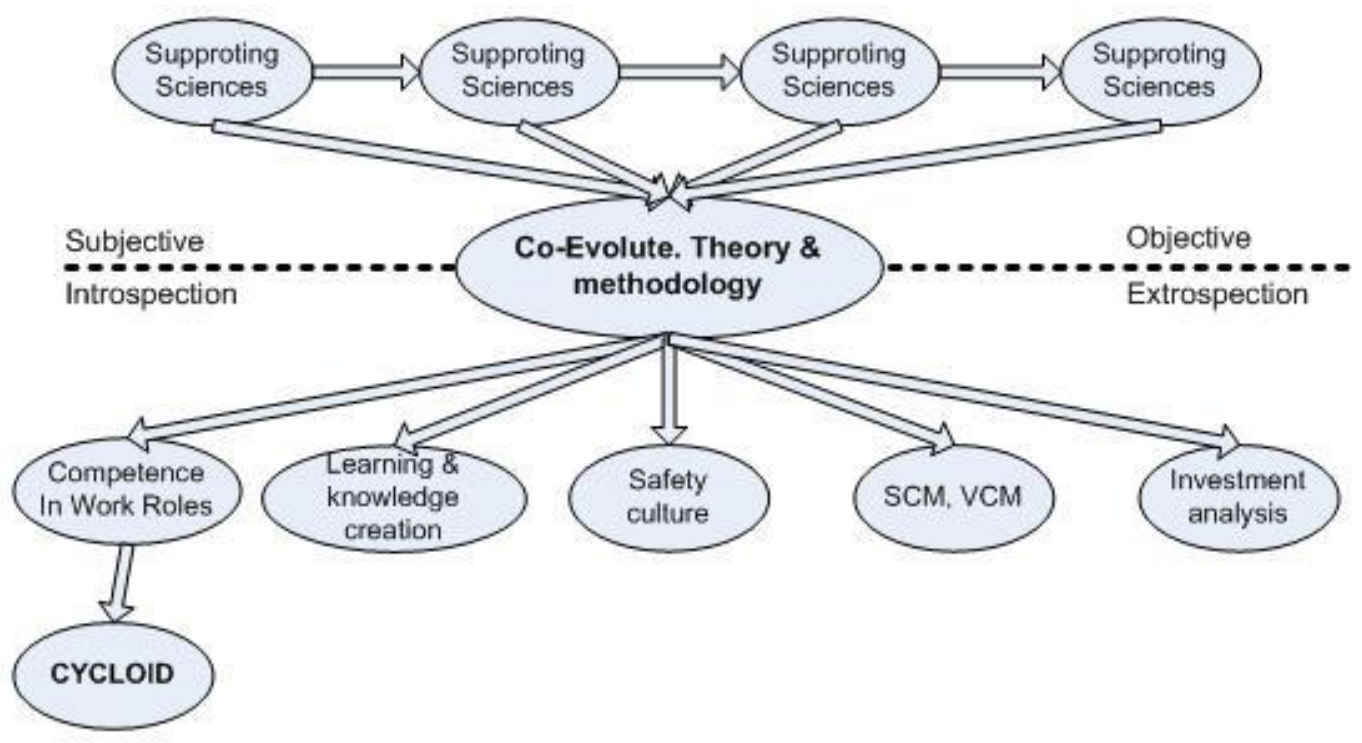

Fig. 1: Modelo Evolute: teoría, metodología y aplicaciones Fuente: Copyright 2005:(Vanharanta, 2005)

La herramienta contiene 30 competencias agrupadas en 6 grupos (fig. 2 y 3): autoconocimiento, autocontrol, capacidad cognitiva, automotivación, empatía y habilidades sociales. Estos 6 grupos se engloban en otros dos: las competencias personales y las competencias sociales. Este modelo está pensado para puestos de trabajo genéricos, haciendo hincapié en un gestor de proyectos, definiendo a la persona como responsable en la gestión de trabajos complejos, con capacidad de interactuar en colaboración con otras personas. El proceso seguido consiste en la autoevaluación del alumno. Este arquetipo lo encontramos muy adecuado para evaluar estudiantes, porque se pueden considerar gestores de su propio proyecto de aprendizaje, es decir, sus estudios (Makatsoris, 2009; Müller y Turner, 2010).

Los resultados de la autoevaluación tienen como objetivo (Nurminen, 2003) la identificación de puntos débiles y por tanto la necesidad de formación, la evaluación del potencial de una persona y la evaluación del rendimiento, generando efectos sobre la motivación y las decisiones sobre la propia carrera formativa o profesional. La eficacia de la autoevaluación depende de varios factores: el contenido de la evaluación, el método utilizado y la cultura de la organización. La autoevaluación, se considera un método eficiente para el desarrollo personal, para clarificar roles y para comprometer los objetivos de un proyecto (Nurminen, 2003).

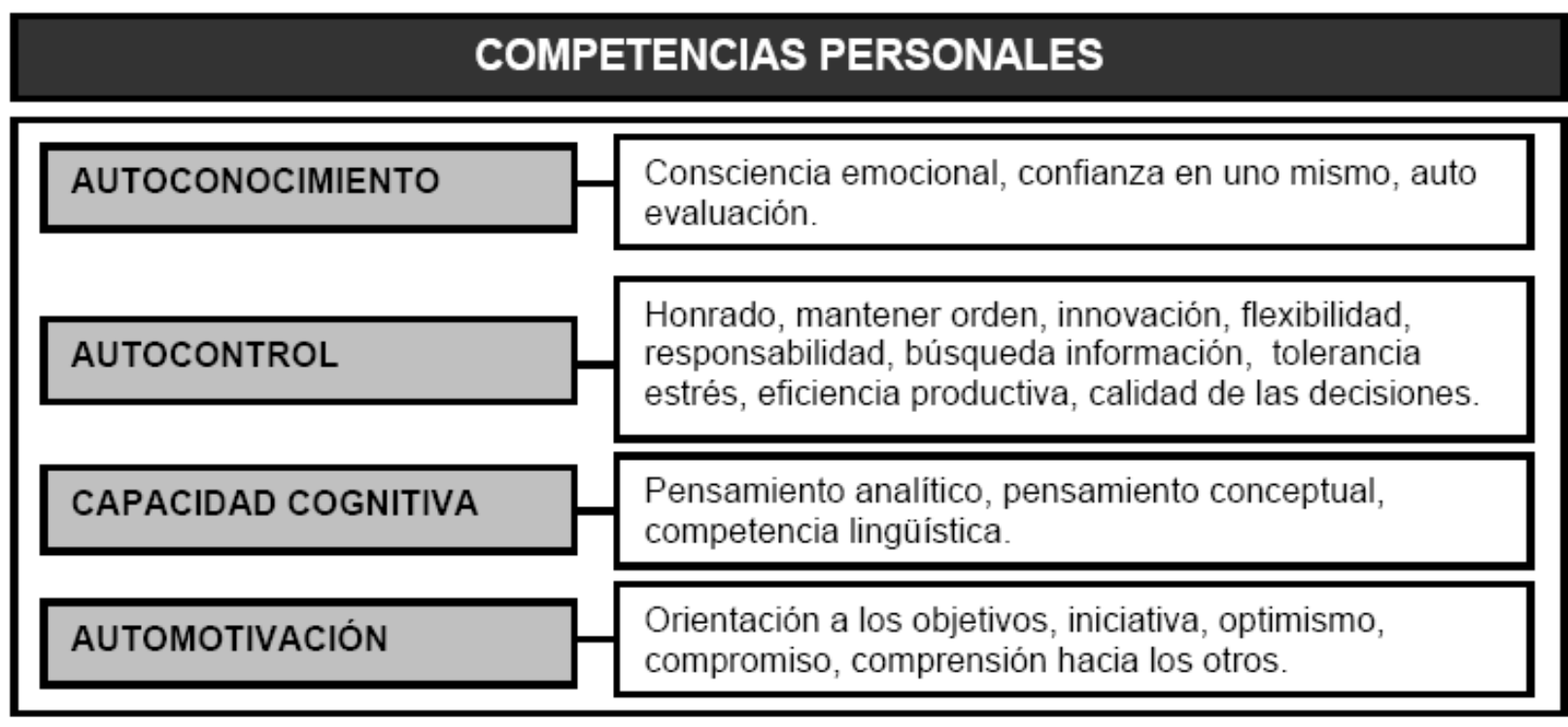

Fig.2: Modelo de competencias personales Cycloid 


\section{COMPETENCIAS SOCIALES}

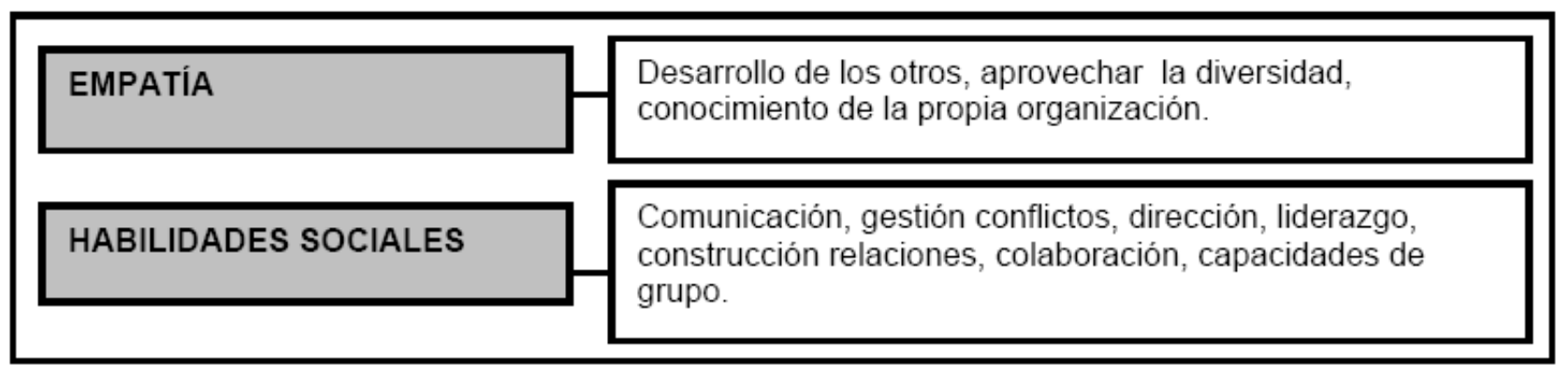

Fig.3: Modelo de competencias sociales Cycloid

Aunque no está libre de inconvenientes, como ejemplo, se observa la tendencia de las personas a evaluar sus propios resultados mejor que los de los demás. También cabe destacar, que el grado de precisión de la autoevaluación varía entre estudiantes (Cronbach, 1990), por ejemplo, las personas tienen capacidades dispares de observación, de autocrítica, de madurez y motivaciones diferentes. Para una misma persona la autoevaluación puede variar en el tiempo, debido al crecimiento mental o personal, al aprendizaje y a los cambios de personalidad o salud, sin embargo, aunque los alumnos se evalúan con precisión, las ventajas de la autoevaluación son de gran ayuda para analizar sus capacidades (Bikfalvi et al., 2007).Para resolver en parte esta situación, se plantea la evaluación como un cotejo entre distintas competencias, más que una comparación con el rendimiento de otras personas. Este proceso permite un enfoque de mejora continua (Makatsoris, 2009).

Las competencias se evalúan de forma indirecta ya que se puntúan afirmaciones relacionadas con el trabajo diario de las personas. El alumno no tiene la impresión que se evalúa su rendimiento, sino su actitud, manera de actuar, sentimientos, etc...Para cada competencia, por cada afirmación que hay que valorar, el alumno medirá su nivel actual y el nivel que considera debería tener, porque lo necesita o porque lo desearía tener. Este nivel, sólo se puede tener mediante la autoevaluación, porque quien conoce mejor el nivel de aspiraciones y objetivos de una persona es normalmente ella misma. La diferencia entre estos dos niveles, el necesario o deseado y el actual, es lo que llamamos tensión creativa. La tensión creativa, permitirá identificar competencias donde hay una percepción de necesidad de aprendizaje. La autoevaluación, la autocrítica y la conciencia, son la base del aprendizaje reflexivo. La tensión creativa de cada alumno es única (Kantola et al., 2005). En líneas generales podemos afirmar que los estudiantes son capaces de reconocer sus características personales a partir de los resultados del estudio (Chang, 2007).

Las características diferenciadoras de esta aplicación, independientemente de su carácter TIC, las podemos concretar en tres puntos principales:

i) Se basa en una especificación explícita de acuerdo con una conceptualización del dominio de competencias del puesto de trabajo de un gestor de proyectos (Jonassen, 2006).

ii) Incluye los niveles necesarios para las competencias y esto permite identificar la tensión creativa de las personas.

iii) La utilización de soluciones de lógica borrosa (fuzzy logic) (Zadeh, 1965), permite que los alumnos puedan valorar libremente su nivel de competencias actuales y sus aspiraciones, dentro de una escala semántica continua.

Cycloid, como todas las aplicaciones basadas en las TIC, tiene la ventaja de producir un retorno instantáneo al alumno al autoevaluarse, así como generar información instantánea al centro y profesorado. El objetivo de esta herramienta es complementar otros posibles instrumentos, ayudando a construir una imagen lo más completa posible sobre las características únicas de cada alumno, para descubrir de esta manera, las carencias percibidas y las necesidades de formación. Es por ello una aplicación que coincide en gran parte con los objetivos propuestos en nuestra universidad. 


\section{METODOLOGÍA}

El estudio lo hemos realizado en dos etapas claramente diferenciadas: pre-evaluación y evaluación, como se describe en lo que sigue.

\section{Pre-evaluación:}

Consistente en informar a los alumnos sobre el contenido y el funcionamiento de la herramienta. Se les envía una guía donde se explica la aplicación y su utilidad, proporcionando explicaciones sobre las competencias sociales y personales. El acceso a la aplicación se realiza a través de una contraseña. Se les explica igualmente que tienen que pensar desde la posición de un gestor de proyectos, ya que la aplicación que utilizamos está pensada en esa dirección. En nuestro caso un gestor de proyectos abarca un ámbito muy general, es por eso que hacemos que el estudiante vea sus estudios como la realización de un proyecto.

\section{Evaluación:}

Los estudiantes deben responder 120 afirmaciones relativas a cuestiones cotidianas, donde se debe valorar el nivel actual y el deseado. Estas afirmaciones engloban los seis grupos de competencias. Por ello, se utiliza una escala de medición que contiene etiquetas lingüísticas que gradúan si la consideración es: siempre, a menudo, a veces o nunca (Kantola et al., 2005).El tiempo de la autoevaluación es de aproximadamente unos 50 minutos, obteniendo al final un informe gráfico individual sobre el nivel de competencias actual, así como el deseado y el correspondiente a la tensión creativa, donde se puede observar de forma clara donde se encuentran las grandes diferencias entre los niveles actuales y los deseados. Los gráficos se pueden obtener de forma inmediata en formato de diagramas de barras o gráfico de telaraña.

\section{RESULTADOS}

\section{Estudio curso 2007-2008}

Este estudio fue realizado mediante la aplicación, complementado con un cuestionario de postevaluación. Los estudiantes que participaron en las dos partes del proceso fueron: el curso 20072008, alumnos de primero de DI con un total de 24 estudiantes y otro grupo de primero de EA con 11 estudiantes. Hemos seleccionado un gráfico de barras que refleja el orden de las competencias con una tendencia decreciente en tensión creativa. Optamos por ésta porque nos da una diferenciación clara entre cuál es la situación actual y cuál es el objetivo de futuro del alumno. Este último aspecto puede ser considerado un punto importante de partida para un estudiante. Nuestro reto consistió en analizar cuáles eran las diferentes competencias que los alumnos seleccionaban como más importantes a desarrollar, por tanto, su objetivo, y cuáles eran por consiguiente, las percepciones más importantes en competencias en su actual situación. La tensión creativa nos indica el desarrollo personal y los planes de los estudiantes en un futuro.

En los resultados para cada grupo obtuvimos un gráfico de barras (fig. 4) donde el color azul nos indicó el estado actual y el color rojo cuál es era el objetivo. Lo más destacable en ambos casos, es que la tolerancia al estrés es el concepto principal a mejorar independientemente de la especialidad. El mismo caso lo encontramos en Chang (2009). En un segundo nivel, encontramos diferencia entre los alumnos de DI, que designan la calidad de las decisiones como aspecto a mejorar y los alumnos de EA para quines la competencia lingüística es una capacidad importante, y por tanto aspecto deseado a conseguir.

A partir de estos datos, nuestro objetivo ha consistido en buscar cuál es el camino para introducir estas competencias en los programas de estudio. Un ejemplo ha sido en el caso de la competencia lingüística, la introducción de textos en lengua inglesa. En otro sentido, la agrupación de estas treinta competencias en grupos de seis, nos dio un resultado en donde los estudiantes de los dos grupos destacaron la competencia de autocontrol, como motivo de mejora. 
Evolute / Cycloid / Competencias / Tensión creativa

Tolerancia al estrés

Calidad de las decisiones

Pensamiento conceptual

Innovación

Comunicación

Conocimiento de la propia organización

Capacidades de grupo

Comprensión de los demás

Flexibilidad

Eficiencia productiva

Construcción de relaciones

Autoevaluación

Competencia lingüística

Búsqueda de información

Pensamiento analítico

Responsabilidad

Diversidad de influencias

Compromiso

Dirección

Conciencia emocional

Optimismo

Gestión de conflictos

Mantenimiento del orden

Honradez

Confianza en uno mismo

Iniciativa

Liderazgo

Desarrollo de los demás

Orientación a los objetivos

Colaboración

Evolute / Cycloid / Competencias / Tensión creativa

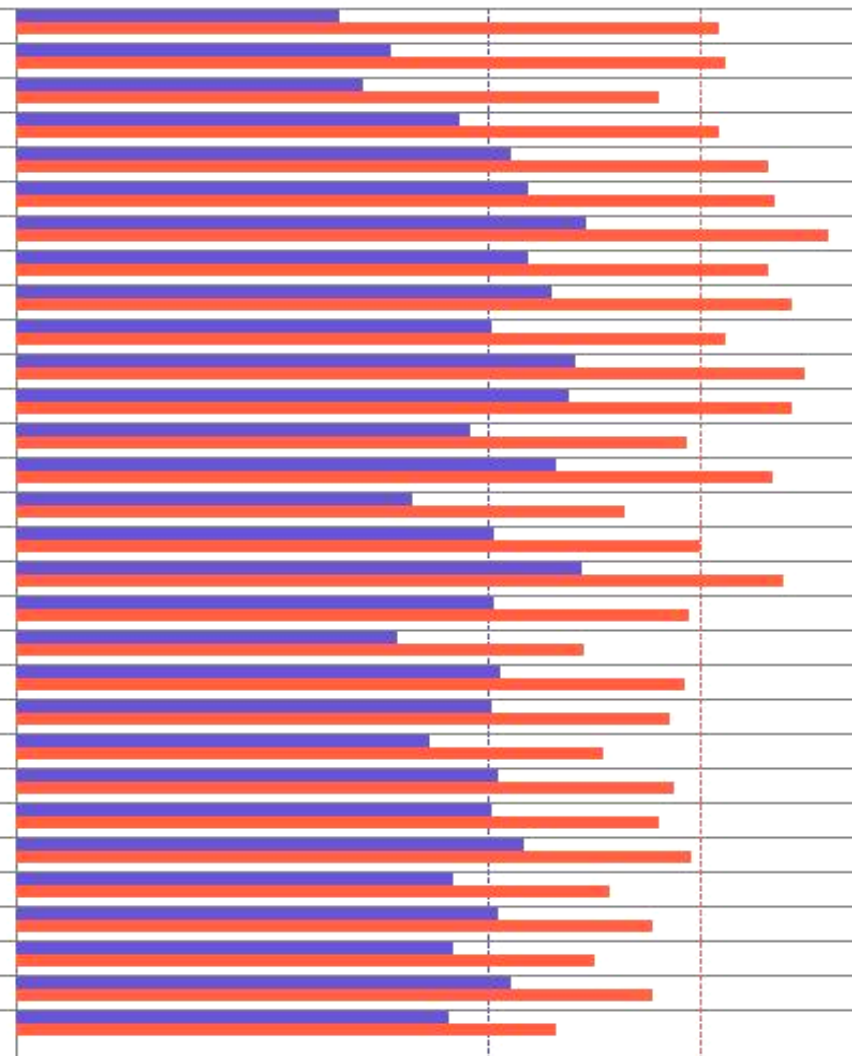

DI24 estudiantes

Tolerancia al estrés

Competencia lingüística

Flexibilidad

Mantenimiento del orden

Optimismo

Autoevaluación

Comunicación

Orientación a los objetivos

Calidad de las decisiones

Búsqueda de información

Confianza en uno mismo

Liderazgo

Innovación

Capacidades de grupo

Comprensión de los demás

Construcción de relaciones

Eficiencia productiva

Conocimiento de la propia organización

Desarrollo de los demás

Compromiso

Responsabilidad

Dirección

Honradez

Pensamiento analítico

Conciencia emocional

Iniciativa

Pensamiento conceptual

Gestión de conflictos

Colaboración

Diversidad de influencias

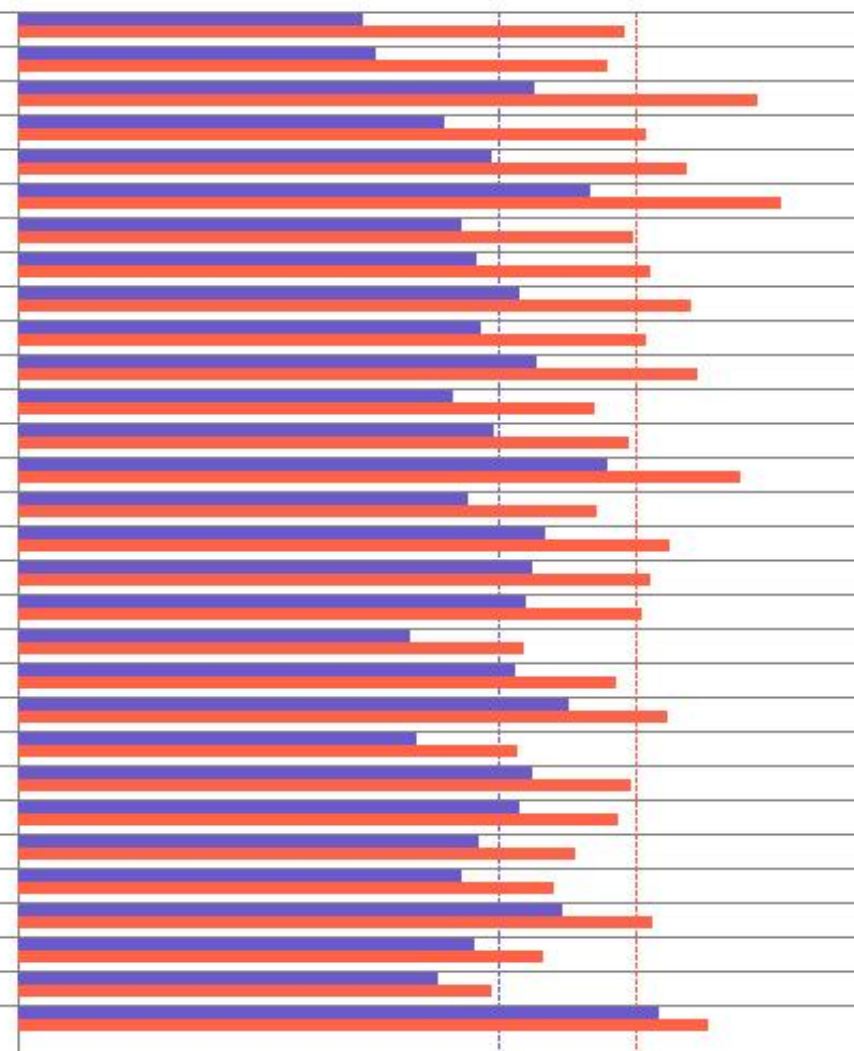

EA 11 estudiantes

Fig.4: Tensión creativa alumnos de primer curso 07/08 


\section{Estudio curso 2008-2009}

El mismo estudio fue realizado el curso siguiente 08/09 con el primer curso de alumnos de DI con 16 estudiantes y el primero de EA con 13. Lo más destacable fue la tolerancia al estrés, en donde se observa una mayor tensión creativa, por tanto, la competencia a mejorar. En un segundo nivel, ambos grupos coincidieron en mejorar la competencia lingüística. Del agrupamiento de treinta a seis competencias, lo más significativo para el grupo de alumnos de DI fue la competencia de autocontrol, y para los alumnos de EA la capacidad cognitiva. Respecto al agrupamiento de las seis competencias en dos grupos principales, las personales y las sociales, todos los grupos marcaron la competencia personal como la que más debían de mejorar, por encima de la social.

\section{Resultados cualitativos de tendencia y dispersión de los resultados}

Con la intención de obtener más información y ayudarnos a interpretar mejor los resultados, elaboramos un gráfico con los datos de dos años para poder analizar la dispersión de las treinta competencias. Este gráfico está separado por especialidades para encontrar los puntos más coincidentes en cada una y de esta manera analizar los cambios para emprender mejoras.

\section{Grupo de DI}

Detectamos diferencias (fig. 5) entre algunas competencias de los dos años del estudio: tolerancia al estrés y eficiencia productiva. Mientras que en el primer caso se trata del aspecto donde el grado de mejora puede ser más evidente debido a la coincidencia de los dos grupos, encontramos el segundo en donde la valoración por parte de los dos grupos no es concordante, por tanto menos evidente.

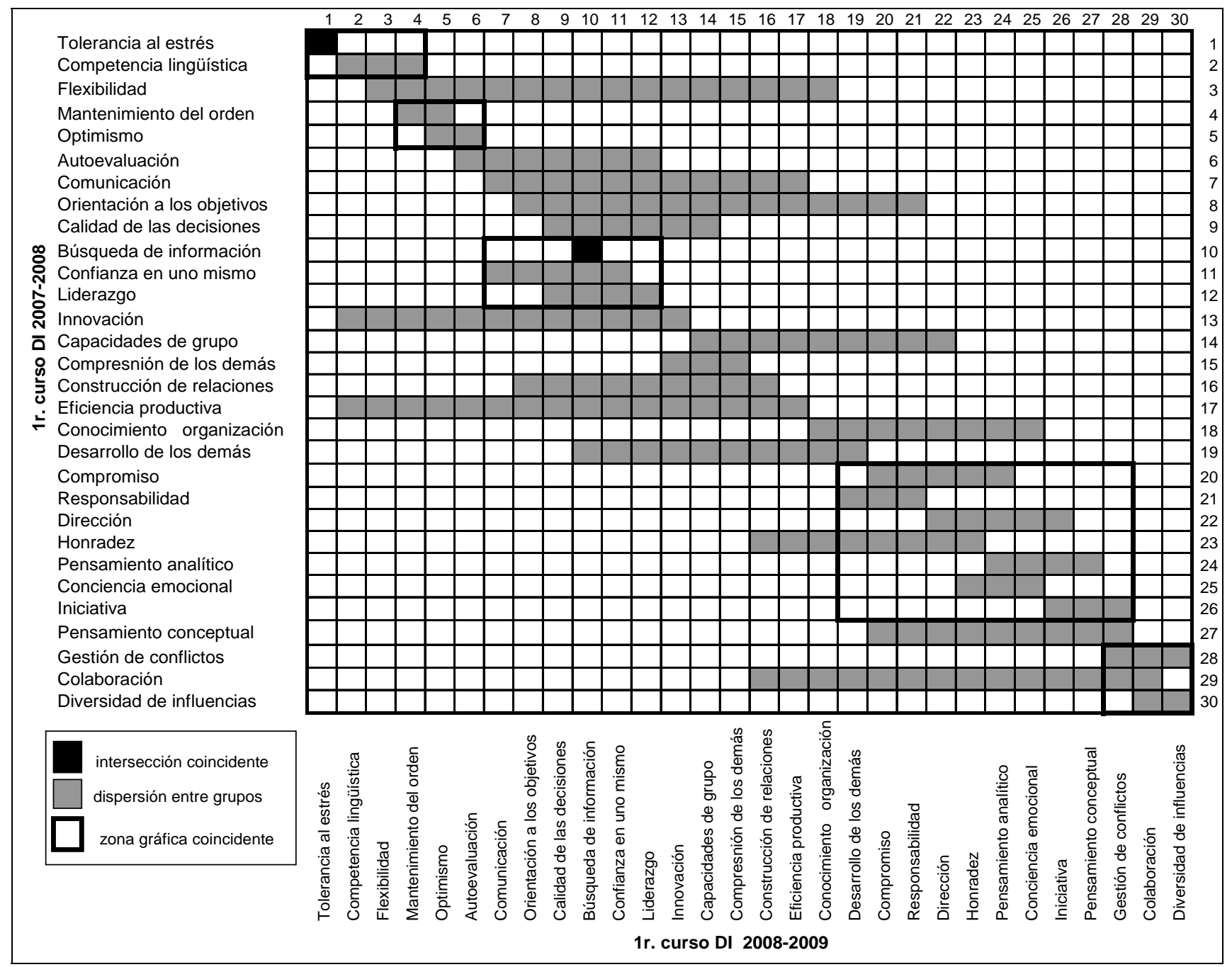

Fig.5: Dispersión en tensión creativa 1r. Curso de DI (07/08 y 08/09) 
Los rectángulos de línea gruesa, marcan un área o zona donde los dos cursos son más coincidentes, en este caso, el primer rectángulo muestra la tolerancia al estrés y la calidad en las decisiones. Nuestra propuesta de mejora para el presente curso ha consistido en organizar el trabajo en grupos al azar, intentando evitar la formación de grupos de amigos. En el segundo rectángulo, destaca la capacidad de innovación (actitud abierta hacia nuevas ideas, opiniones e información) y la comunicación (escucha sincera y envío de mensajes). Este marco nos indica que los estudiantes están interesados en cambiar, pero menos que en el primer caso. La competencia de las comunicaciones, en nuestra universidad, se trabaja en la presentación de proyectos y la competencia en la innovación se integra en el desarrollo de temas y actividades propias de nuestra asignatura. En el centro del gráfico, coinciden los dos grupos de alumnos en resaltar las competencias de eficiencia de producción (realizar tareas de forma rápida y eficaz). Los dos últimos rectángulos, están compuestos por el optimismo, la gestión de conflictos, la confianza en uno mismo, la iniciativa, el desarrollo de otros y la colaboración.

A partir de estos resultados, nuestro departamento ha tomado la decisión de trabajar en más profundidad las competencias en ética de la responsabilidad, el optimismo, la gestión de conflictos y la confianza en sí mismo, introduciéndolas con una mayor importancia en los proyectos que desarrollan en las distintas asignaturas de los estudios.

\section{Grupo de EA}

Con idéntico objetivo que en el análisis anterior, hemos realizado el mismo estudio con los cursos 07/08 y 08/09 de los alumnos de EA para poder analizar en el gráfico de dispersión la tensión creativa de las competencias (fig. 6). En esto segundo gripo de estudiantes se detectan algunas coincidencias y diferencias. La primera coincidencia y más remarcable es, como viene siendo habitual en todo el estudio, la tolerancia al estrés y la competencia lingüística. En el presente curso académico la mejora que hemos introducido en algunas asignaturas ha consistido en la inserción de textos y comentarios en lengua inglesa, así como la posibilidad de presentar algunos proyectos en esta lengua.

Igualmente hemos podido observar las competencias correspondientes a la autoevaluación y el optimismo como coincidentes. Con el objetivo de solucionar en parte este problema, la mejora que hemos introducido este curso ha sido la introducción en la asignatura de expresión gráfica de trabajos autoevaluables, siguiendo una tabla de revisión y evaluación definida. Queda claro que estas actuaciones son criticables y veremos con el tiempo si han producido alguna mejora.

En el tercer grupo, la búsqueda de información, la propuesta ha sido la inclusión en alguno de los bloques del temario de un ejercicio de búsqueda de información, utilizando las TIC. En medio de la tabla destacan las competencias de la innovación, la eficiencia productiva y la orientación por objetivos, donde hemos adaptado las mismas mejoras comentadas en la figura anterior.

\section{CONCLUSIONES}

Del estudio presentado se pueden deducir las siguientes conclusiones:

i) La convergencia entre los deseos de los estudiantes y la demanda del mundo profesional debería estar entre los objetivos de cualquier centro de educación superior.

ii) Cycloid ofrece información al profesorado sobre sus estudiantes evaluando en qué estado se encuentran los alumnos en algunas de las competencias transversales y analizando si son cubiertas, pudiéndose trabajar en un proceso continuo de aprendizaje. Para los profesores este proceso permite la adopción de mejoras continuas ya que refleja con rapidez los resultados pudiendo modificar los programas y contenidos de las asignaturas. Puede ser una herramienta de ayuda para mejorar la calidad en la enseñanza introduciendo medidas que disminuyan la tensión creativa en las competencias con mayores problemas.

iii) La aplicación utilizada fomenta un aprendizaje más colaborativo, al poder ayudar el centro y profesorado en particular a mejorar las competencias con una diferencia en tensión creativa mayor. 


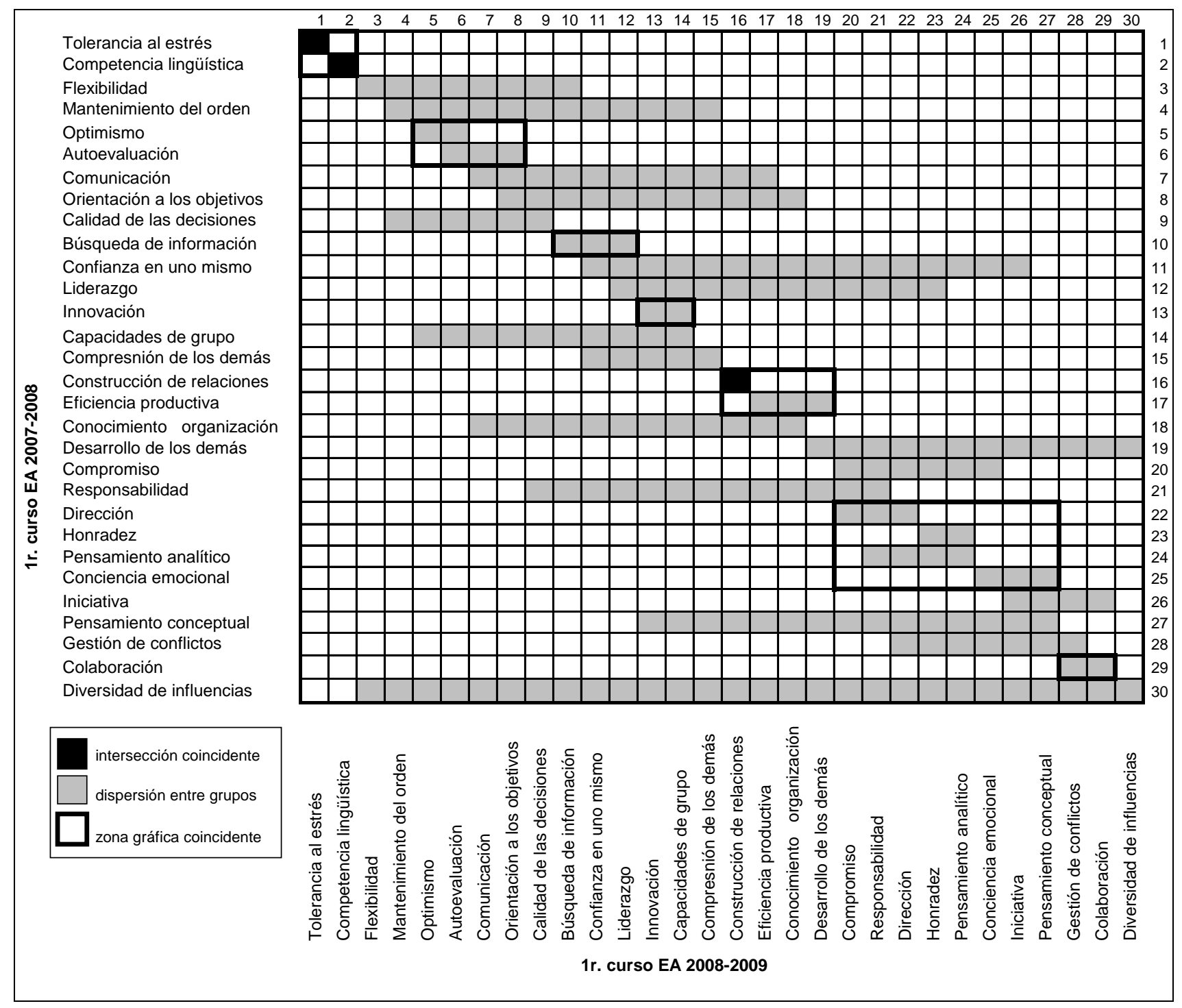

Fig.6: Dispersión en tensión creativa 1r. Curso E A (07/08 y 08/09)

iv) La aplicación genera un aprendizaje más constructivo al poder el estudiante observar su estado actual y objetivos personales, reconociendo sus puntos fuertes y débiles, siendo esta tensión creativa esencial para el desarrollo personal, pudiendo reorientar sus estudios. La autoevaluación permite a los estudiantes conocerse mejor y por tanto acercarse a los objetivos propuestos en las competencias transversales.

v) Los estudiantes reconocieron sus características personales de acuerdo a los resultados obtenidos. El resultado individual de su nivel puede ser útil en el diseño y desarrollo de estrategias para afrontar mejor sus estudios y trabajo como ingenieros.

\section{REFERENCIAS}

Bikfalvi, A., Pagés, J. L., Kantola, J., Marqués, P. y Mancebo, N. Complementing education with competence development: an ICT-based application. International Journal of Management in Education. Vol.1, no 3, 231-250 (2007)

Chang, Y., Kantola, J. y Vanharanta, H., A Study of Creative Tension of Engineering students in Korea, Human Factors and Ergonomics in Manufacturing, Vol. 17 (6) 511-520 (2007)

Chang, Y. , Eklund, T., Kantola, J. y Vanharanta, H., International Creative Tension, Study of University students in South Korea and Finland, Human Factors and Ergonomics in Manufacturing, Vol. 19 (6) 528-543 (2009) 
Coll, R. y Zegwaard, K., Perceptions of desirable graduate competencies for science and technology new graduates, Research in science and Technological Education, Vol. 24, $\mathrm{n}^{\circ} 1,29-58$ (2006)

Cronbach, L.J., Essentials of psychological testing, 5 ed. Harper \& Row, New York (1990)

International Project Management Association. ICB-IPMA competence Baseline. Ed. IPMA, Nijkerk, Netherlands, Version 3.0 (2006)

Jonassen, D., Accommodating ways of human knowing in the design of information and instruction. International Journal of Knowledge and Learning, 2(3-4), 181-190 (2006)

Kantola, J., Vanharanta, H. y Karwowski, W., The evolute system: A co-evolutionary human resource development methodology. In International encyclopedia of human factors and ergonomics, CRC Press, W.Karwowski Ed., 2894-2900 (2005)

Makatsoris, Ch., An Information and Communication Technologies-Based Framework for Enhancing Project Management Education through Competence Assessment and Development, Human Factors and Ergonomics in Manufacturing, Vol. 19 (6) 544-567 (2009)

Mäkiniemi, P., Kantola, J. y Vanharanta, H., Conchoid- A self-evaluation tool for assessing vocational competences of maintenance personnel. 9th International Haamaha Conference: Human \& Organizational Issues in the Digital Enterprise, NUI, Galway, Ireland (2004)

Müller, R. y Turner, R., Leadership competency of successful project managers, International Journal of Project Mangement, 28, 437- 448 (2010)

Nurminen, K., Deltoid- the Competences of Nuclear Power Plant Operators, Master of Science thesis, Finland: Tampere Univesity of thecnology at Pori (2003)

Reichert, S. y Tauch, C., Trends IV: European Universities Implementing Bologna (2003) http://www.eua.be/eua/jsp/en/upload/Trends2003final.1065011164859.pdf, consultado octubre 2008.

Vanharanta, H., Co-evolutionary design for human-compatible systems, International Conference on Computer-Aided Ergonomics, Human Factors and Safety, Kos\&caronice, Las Vegas, USA (2005)

Zadeh, L.A, Fuzzy Sets, Information and Control, vol. 8 pp. 338-353 (1965) 